\title{
Synthesis and antioxidant activity of newly synthesized chalcones
}

\author{
Milica Tasić ${ }^{1 *}$, Nemanja Turković ${ }^{2}$, Branka Ivković ${ }^{1}$, Jelena Kotur-Stevuljević ${ }^{3}$, \\ Zorica Vujic ${ }^{1}$ \\ ${ }^{I}$ Department of Pharmaceutical Chemistry' Faculty of Pharmacy, University of Belgrade, 11000 Belgrade, Serbia \\ ${ }^{2}$ Agency for Medicines and Medical Devices of Montenegro, 81000 Podgorica, Montenegro \\ ${ }^{3}$ Department of Medical Biochemistry' Faculty of Pharmacy, University of Belgrade, 11000 Belgrade, Serbia
}

\section{Introduction}

Chalcones (1,3-diaryl-2-propen-1-ones) are natural products abundant in fruits, vegetables, spices and teas. More recently, chalcones are obtained synthetically by the reaction of aldol condensation (Claisen-Schmidt condensation). Chalcones have a wide range of biological activities: antiinflammatory, antioxydant (Halliwell and Gutteridge, 2006), antibacterial, antifungal, antiproliferative (Dimmock et al., 1998) and others, as well as low selectivity that limits their clinical application.

\section{Materials and methods}

Synthesis of chalcones

Compounds used in synthesis were mono- and disubstituted halogenated benzaldehyde which gave the corresponding chalcones in the reaction with the mono- or disubstituted acetophenone. Chalcones were synthesized in the reaction of base catalysed aldol condensation.

\section{Structural analysis of synthesized substances}

The structures of compounds were determined by infrared spectroscopy (IR), nuclear magnetic resonance (NMR) and mass spectroscopy (MS). Melting temperatures of newly-synthesized chalcone derivatives were determined by the instant melting method.

\section{Antioxidant activity assay}

The antioxidant activity was tested in serum of healthy adults by determining the following parameters: TAS (total antioxidant status), TOS (total oxidative status), $\mathrm{PAB}$ (prooxidative antioxidant balance), SHG (sulfhydryl group content) (Erel, 2005). Based on these values, Zscores of protoxidative (TOS+PAB) and antioxidative (TAS+SHG) parameters were calculated, and from their difference, an oxidative score was obtained as a measure of antioxidant activity. An oxidative score was also determined in serum samples in which except chalcones, the tertbutyl hydroperoxide (TBH), strong synthetic prooxidant was added and in pure serum as well.

\section{Results and discussion}

Chalcones were synthesized by Claisen-Schimdt condensation and after their physicochemical characterization 18 derivatives of chalcones that showed a high degree of purity after purification

\footnotetext{
*milicatasicdj@hotmail.com
} 
were selected and were tested for antioxidant activity. When the oxy scores were calculated for all compounds, the obtained values were statistically processed in the SPSS program.

The oxidative score of certain chalcones was in range of 9.56 to 283.67 (133.93 \pm 83.06 ), while for the group of chalcones containing two phenol groups in its structure, oxidative score was in range of -44.07 to $-151.62(-89.13 \pm 40.87)$.

As the oxy score is an indicator of strength, i.e. capacity of an antioxidant activity of a compound, compound having a negative value of this score will exhibit significant antioxidant activity. Such values were observed in the second group of compounds which differ from the first group in terms of structure in the presence of another phenolic group in the ortho position. This is confirmation of previous studies where hydroxy chalcones have been shown to have a potent inhibitory effect on the formation of superoxide anions in rat's neutrophils (Ni et al., 2004).

The steric influence of the substituents was also shown (Kim et al., 2008) and the substituents in the ortho and para positions had a negative effect on the antioxidant value as opposed to the substituents in the meta position.

As demonstrated in the study, electron-attracting groups reduce the efficiency of hydrogen release from the amino group of pyrazolines and therefore reduce the antioxidant activity (Akshay et al., 2013).

\section{Conclusion}

Analyzing of all obtained data, it is concluded that chalcones have antioxidant activity whose potential depends on the substituents as well as their position in the molecule (Batovska, 2010).
ANOVA test showed a statistically significant difference between the oxidative scores of the group of chalcones with one and two phenol groups $(\mathrm{p}<0.05)$, what indicates that there is a significant influence of the presence of several phenol groups in the compound on the antioxidative activity of chalcones.

Besides this, substituents such as halogens and other electron distractors should be avoided. Meta position of the substituent has the highest contribution to the antioxidant activity.

\section{References}

Akshay, K., Varadaraj, B.G., Singla, R.K., 2013. Synthesis and evaluation of antioxidant activity of novel 3,5-disubstituted-2-pyrazolines. Bull. Fac. Pharm. Cairo Univ. 51(2), 167-173.

Batovska, D.I., 2010. Trends in utilization of the pharmacological potential of chalcones. Curr. Clin. Pharmacol. 5, 1-29.

Dimmock, J.R., Kandepu, N.M., Hetherington, M., Quail, J.W., Pugazhenthi, U., Sudom, A.M., Chamankhah, M., Rose, P., Pass, E., Allen, T.M., Halleran, S., Szydlowski, J., Mutus, B., Tannous, M., Manavathu, E.K., Myers, T.G., De Clercq, E., Balzarini, J., 1998. Cytotoxic activities of Mannich bases of chalcones and related compounds. J. Med. Chem. 41(7), 10141026.

Erel, O., 2005. A new automated colorimetric method for measuring total oxidant status. Clin. Biochem. 38, $1103-1111$.

Halliwell, B., Gutteridge, J.M.C., 2006. Free radicals in biology and medicine. Clarendon Press Oxford Ed. 4

Kim, B.T., Chun, J.C., Hwang, K.J., 2008. Synthesis of dihydroxylatedchalcone derivatives with diverse substitution patterns and their radical scavenging ability toward DPPH free radicals. Bull. Korean Chem. Soc. 29(6), 1125-1130.

Ni, L., Meng, C.Q., Sikorsk J.A., 2004. Recent advances in therapeutic chalcones. Expert Opin. Ther. Patents 14(12), 1669-1691. 